\title{
Indication for endoscopic treatment based on the risk of lymph node metastasis in patients with Siewert type II/III early gastric cancer
}

\author{
Jeung Hui Pyo ${ }^{1} \cdot$ Hyuk Lee ${ }^{2}$ ( $)$ Yang Won $\mathrm{Min}^{2} \cdot$ Byung-Hoon Min $^{2} \cdot$ Jun Haeng Lee ${ }^{2} \cdot K_{\text {Koung-Mee Kim }}^{3}$. \\ Heejin $\mathrm{Yoo}^{4}$. Soohyun Ahn ${ }^{4}$. Jae J. Kim ${ }^{2}$
}

Received: 8 June 2017 / Accepted: 7 December 2017 / Published online: 14 December 2017

(c) The International Gastric Cancer Association and The Japanese Gastric Cancer Association 2017

\begin{abstract}
Background Because of the poor prognosis of proximal gastric cancers, there is debate as to whether the conventional indications for endoscopic resection can be used.

Methods Among subjects who underwent surgery for esophagogastric junction or gastric cardia cancer, 256 patients with T1 type II/III of the Siewert classification were included in this study. The association of lymph node metastasis (LNM) with each variable was analyzed using logistic regression models. A receiver operating characteristic curve was used to determine the discriminatory ability of the model. Propensity score-matched non-cardia cancer patients were selected to compare LNM and long-term survival rates.

Results Of the 256 patients with T1 Siewert II/III gastric cancer, 21 (8.2\%) had LNM. Because there was no LNM in T1a cancers, risk factors were analyzed only in patients with T1b. Tumor size (OR 1.42, 95\% CI 1.10-1.82, $P=0.007$ ) and lymphovascular invasion (LVI) (OR 5.13, 95\% CI 1.88-14.06, $P=0.002$ ) were determined to be predictors of LNM $($ sensitivity $=66.7 \%$ and specificity $=81.6 \%)$. Among patients without LVI, the groups with negligible risk for LNM were mucosa-confined cancer, or SM1 cancer with a tumor size $\leq 3 \mathrm{~cm}$. No LNM was observed in patients satisfying the absolute or extended criteria for endoscopic resection of early gastric cancers. LNM and long-term survival rates of patients with Siewert II/III did not differ significantly compared with matched non-cardia cancer patients.

Conclusions Tumor size and LVI were associated with LNM in patients with early Siewert type II/III gastric cancer, and the expanded indication for endoscopic resection may be used.
\end{abstract}

Keywords Early gastric cancer $\cdot$ Siewert type II/III $\cdot$ Endoscopic resection $\cdot$ Lymph node metastasis

Electronic supplementary material The online version of this article (https://doi.org/10.1007/s10120-017-0789-3) contains supplementary material, which is available to authorized users.

Hyuk Lee

leehyuk@skku.edu

1 Center for Health Promotion, Samsung Medical Center, Seoul, Republic of Korea

2 Department of Medicine, Samsung Medical Center, Sungkyunkwan University School of Medicine, Irwon-dong, Gangnam-gu, Seoul 135-710, Republic of Korea

3 Department of Pathology, Samsung Medical Center, Sungkyunkwan University School of Medicine, Seoul, Republic of Korea

$4 \quad$ Statistics and Data Center, Research Institute for Future Medicine, Samsung Medical Center, Seoul, Republic of Korea

\section{Introduction}

Adenocarcinoma originating from the esophagogastric junction (EGJ) has been recently rapidly increasing in incidence in both Western and Eastern countries [1,2]. In addition to histological classification, stratification according to anatomical location has been suggested [3-5]. Gastric cancers of proximal origin (cardia/EGJ) are reported to have poor prognosis when compared with distal cancers (body, antrum) [5]. Carcinomas originating from the proximal region have been reported to have a poor prognosis, with 5-year survival rates of 24-36\% [6, 7]. In 1996, Siewert proposed a classification of EGJ adenocarcinomas, based on their location [8], which has been used by surgeons to guide treatment plans. Type I tumors are true esophageal cancers treated with thoracotomy, whereas type II and III are regarded as equivalent tumors and are surgically approached in this manner $[9,10]$. 
Endoscopic resection of cardia or EGJ cancers has the great advantage of avoiding total gastrectomy, which affects patient quality of life [11-13]. However, there is still uncertainty about the appropriate treatment of early gastric cancer, considering the poor prognosis of proximal gastric cancers as reported in previous studies $[5,6]$. There is debate as to whether the conventional indications for endoscopic resection can be used. To the best of our knowledge, no study has evaluated the feasibility of endoscopic resection of cardia or EGJ cancers based on the status of lymph node metastasis (LNM). There is only limited evidence for the validity of the absolute or expanded indications for endoscopic resection of cardia or EGJ cancers. The evaluation of risk factors that predict the presence of LNM may help identify patients who are at low risk for LNM and would benefit from avoiding an unnecessary total gastrectomy. Therefore, the aim of this study was to evaluate the risk factors associated with LNM and identify patients with a negligible risk of LNM in early Siewert type II and III gastric cancer.

\section{Methods}

\section{Patients}

Patients were selected from the prospective database of the Department of Surgery, Samsung Medical Center between January 2002 and March 2017. Among subjects who underwent surgery for EGJ or cardia cancer, 256 patients with pathologically diagnosed $\mathrm{T} 1$ (confined to the mucosa or submucosal cancer) type II or III according to the Siewert classification [14] were included. Siewert subtypes of EGJ adenocarcinoma were determined based on the findings of an endoscopic examination and the pathological analysis of resected specimens. Type I is defined as tumors with the epicenter located $1-5 \mathrm{~cm}$ above the EGJ regardless of invasion to the EGJ; type II are tumors that invade the EGJ with the epicenter located between $1 \mathrm{~cm}$ above and $2 \mathrm{~cm}$ below the EGJ; and type III are tumors that invade the EGJ, with the epicenter located $2-5 \mathrm{~cm}$ below the EGJ.

In our institution, Siewert II/III gastric cancer is treated based on the Japanese Gastric Cancer Treatment Guidelines [15]. Early gastric cardia cancers meeting the absolute criteria (differentiated mucosal cancer without ulceration, and $\leq 2 \mathrm{~cm}$ based on gross endoscopic findings) will undergo endoscopic resection and those beyond the criteria will be candidates for surgery. However, according to the post-endoscopic resection pathological findings, those meeting the expanded criteria (SM1 and $\leq 3 \mathrm{~cm}$, or an ulcerative mucosal lesion $>3 \mathrm{~cm}$ ) will be monitored, whereas those beyond the expanded criteria will undergo additional surgery. Because the location of these cancers makes endoscopic resection challenging, the feasibility of such a procedure is determined by an endoscopist with at least 5 years of experience.

The clinicopathological factors examined included age, sex, tumor location, macroscopic appearance, size, invasion depth, presence of lymphovascular invasion (LVI), and presence of LNM. Macroscopic appearance of the tumor was classified into four types: elevated (I, IIa, I + IIa, IIa + IIb), flat (IIb), depressed (IIc, IIc + III, III), and mixed (other). Submucosal invasion was divided into "upper third" (SM1 $<500 \mu \mathrm{m}$ from the muscularis mucosae), "middle third" (SM2), and "lower third" (SM3). R0 resection was defined as resection of the tumor without histological evidence of cancer in the lateral or vertical margins. Overall survival was determined from the date of surgery to death, disease-specific survival from the date of surgery to death from gastric cancer, and recurrence-free survival from the date of surgery to first relapse. This study was approved by the Institutional Review Board of Samsung Medical Center.

\section{Surgical procedure}

Total gastrectomy was the most common surgery performed, with proximal gastrectomy performed rarely after 2008. The extent of lymph node dissection (D1+, D2, and D2+) was based on the Japanese Gastric Cancer Treatment Guidelines [15]. D2 or D2+ was performed whenever nodal involvement was suspected. Roux-en-Y reconstruction was preferentially performed after total gastrectomy, with esophagogastrostomy being performed after proximal gastrectomy.

\section{Histopathological evaluation}

The tumor specimens were fixed in $10 \%$ formalin and then cut into 5- $\mu \mathrm{m}$ sections, embedded in paraffin blocks, and stained with hematoxylin and eosin. Complete histopathological examination was performed by expert pathologists. Histologically, tumors were classified into either differentiated (including papillary, well-differentiated, or moderately differentiated tubular adenocarcinoma) or undifferentiated (poorly differentiated adenocarcinoma or signet ring cell carcinoma) types. Early gastric cancers with components of both differentiated and undifferentiated carcinoma were classified according to the quantitatively predominant histological type [16]. To identify LNM, lymph nodes were cut into two pieces, with the cut surface being examined with hematoxylin and eosin staining. Tumors were staged according to the American Joint Committee on Cancer Staging Manual (7th edition) [17].

\section{Statistical analysis}

The primary endpoint was LNM. Continuous variables were reported as mean \pm standard deviation $(\mathrm{SD})$, and categorical 
variables were presented as percentages. Differences between continuous variables were compared using the Wilcoxon rank-sum test, whereas differences between categorical variables were analyzed using the Fisher exact test, and a logistic regression model was used to calculated the odd ratios (OR) and corresponding 95\% confidence intervals (CI) of each potential predictor. Univariate analysis was first calculated for each variable, and then multivariate analysis using backward elimination according to a selection criterion of $P=0.05$. For an unbiased comparison of prognosis, we performed a propensity score-matching analysis between cardia and non-cardia cancer groups. Propensity scores were estimated using a logistic regression model of the following covariates: gastric cancer stage (T1) and patient's age and sex. To compare the LNM rate of patients with cardia and matched non-cardia cancer, the generalized estimating equation was used for data analysis. Only variables with a $P<0.05$ in the univariable analysis were included in the multivariable analysis. To assess the discriminatory ability of the model, a receiver operating characteristic (ROC) curve was used to determine the area under the curve (AUC). For model validation, we drew 1000 bootstrap samples and estimated the bias-corrected ORs and AUCs. Survival in cardia and matched non-cardia cancers was estimated with the Kaplan-Meier method. The association of potential risk factors with long-term outcomes was tested by clustered Cox proportional hazards regression analysis. Here also we only included variables having a $P<0.05$ in the univariable analysis in the multivariable analysis. $P<0.05$ was considered statistically significant. All statistical analyses were conducted using SAS software, version 9.4 (SAS Institute, Cary, NC, USA).

\section{Results}

\section{Baseline characteristics}

Table 1 presents baseline characteristics of patients with Siewert type II and III cancers. Of the 256 patients with T1 Siewert type II/III cancer, $21(8.2 \%)$ had LNM. The mean age of the patients was $60.0(\mathrm{SD}, \pm 9.6)$ years, and a male predominance $(79.7 \%$ ) was observed in the sample. Among the patients, $65.6 \%$ were Siewert type II and $34.4 \%$ were Siewert type III, with the distribution of Siewert type not differing significantly among the LNM-positive and LNMnegative groups $(P=0.092)$. Patients underwent either total gastrectomy $(93.4 \%)$ or proximal gastrectomy $(6.6 \%)$, and all patients had R0 resections. A total of 237 patients $(92.6 \%)$ underwent D2 or more radical lymphadenectomy; the mean number (SD) of lymph nodes retrieved was $41.3( \pm 17.9)$, without a significant difference between those with and without LNM ( $P=0.868)$. The distribution of positive LNM is shown in Online Resource 1. The nodal station showing the highest index was the lesser curvature node (No. 3, 67\%), followed by the left cardia (No. 2, 17\%), left gastroepiploic vessels (No. 4sb, 8\%), left gastric artery (No. 7, 4\%), and the splenic artery (No. 11,4\%). The elevated macroscopic type $(42.9 \%)$ was most prominent in patients with positive LNM, as compared to depressed type $(56.6 \%)$ in patients with no LNM. When compared with negative LNM patients, positive LNM patients had a significantly larger tumor size $(4.8 \pm 3.1$ vs. $3.1 \pm 1.7, P=0.003)$, and the proportion of patients with positive LVI was higher $(57.1 \%$ vs. $13.2 \%$, $P<0.001)$. Among LNM-positive patients, there was no mucosal-confined cancer, and they had a higher proportion of deep submucosal (SM2 or 3 ) cancers (90.4\% vs. $41.3 \%$, $P<0.001)$ when compared to LNM-negative patients.

Furthermore, there was no significant difference in LNM rate between Siewert II/III T1 cancers $(8.1 \%)$ and matched non-cardia T1 cancers $(7.9 \%)$ (OR $0.78,95 \%$ CI $0.37-1.67$, $P=0.528)$ (Online Resource 2).

\section{Risk factor analysis}

Because there was no LNM in mucosa-confined (T1a) gastric cancers, risk factors were analyzed in patients with submucosal invasion (T1b) (Table 2). Univariate analysis showed that patients with positive LNM had a significantly larger tumor size (OR 1.43, 95\% CI 1.14-1.78, $P=0.002$ ), and a higher proportion of positive LVI (OR 5.63, 95\% CI 2.15-14.71, $P<0.001)$, than did subjects without LNM. Depth of invasion showed a borderline level of statistical significance (OR 4.31, 95\% CI 0.96-19.31, $P=0.056$ ). After the multivariate analysis, two predictors remained in the final model: tumor size (OR 1.42, 95\% CI 1.10-1.82, $P=0.007$ ) and LVI (OR 5.13, 95\% CI 1.88-14.06, $P=0.002$ ). From the ROC curve, the multivariate model had an AUC of 0.75 (Fig. 1). Based on the Youden index, the optimal cutoff of the final model was found at a predicted probability $=0.187$, providing a sensitivity of $66.7 \%$ (14 of 21 ) and specificity of $81.6 \%$ (115 of 141). Using the logistic regression equation, the probability of positive LNM status $\left(P_{\mathrm{LNM}}\right)$ is given by

$P_{\mathrm{LNM}}=\frac{e^{(-3.843+0.349 \times \text { size }+1.636 \times \mathrm{LVI})}}{1+e^{(-3.843+0.349 \times \operatorname{size}+1.636 \times \mathrm{LVI})}}$,

where LVI $=1$ if positive and LVI $=0$ if negative, and size is the tumor size. The cutoff of probability at 0.187 yielded the risk of LNM as a function of tumor size and presence of LVI (Fig. 2). The model validation was accomplished with 1000 bootstrap resamples with replacement, and the mean AUC was 0.76 (95\% CI 0.75-0.76) (Fig. 3).

In clinical practice, the presence of LVI in the endoscopically resected specimen indicates a high risk of LNM, and standard surgical resection with nodal dissection is usually recommended. Therefore, we calculated the LNM rates by 
Table 1 Baseline characteristics of patients with Siewert II/III cancer

\begin{tabular}{|c|c|c|c|c|}
\hline & Total $(n=256)$ & $\operatorname{LNM}(-)(n=235)$ & $\operatorname{LNM}(+)(n=21)$ & $P$ value \\
\hline Age (years), mean \pm SD & $60.0 \pm 9.6$ & $60.1 \pm 9.6$ & $58.6 \pm 10.0$ & 0.590 \\
\hline \multicolumn{4}{|l|}{ Sex, $n(\%)$} & 1.000 \\
\hline Male & 204 (79.7) & $187(79.6)$ & $17(81.0)$ & \\
\hline Female & $52(20.3)$ & $48(20.4)$ & $4(19.0)$ & \\
\hline \multicolumn{4}{|l|}{ Siewert type, $n(\%)$} & 0.092 \\
\hline II & $168(65.6)$ & $158(67.2)$ & $10(47.6)$ & \\
\hline III & $88(34.4)$ & $77(35.8)$ & $11(52.4)$ & \\
\hline \multicolumn{4}{|l|}{ Extent of surgery, $n(\%)$} & 1.000 \\
\hline Total gastrectomy & $239(93.4)$ & $219(93.2)$ & $20(95.2)$ & \\
\hline Proximal gastrectomy & $17(6.6)$ & $16(6.8)$ & $1(4.8)$ & \\
\hline \multicolumn{4}{|c|}{ Extent of nodal dissection, $n(\%)$} & 0.938 \\
\hline $\mathrm{D} 1+$ & $19(7.4)$ & $18(7.7)$ & $1(4.8)$ & \\
\hline $\mathrm{D} 2$ & $127(49.6)$ & $117(49.8)$ & $10(7.9)$ & \\
\hline $\mathrm{D} 2+$ & $110(43.0)$ & $100(42.6)$ & $10(9.1)$ & \\
\hline $\begin{array}{l}\text { Number of dissected lymph } \\
\text { nodes, mean } \pm \text { SD }\end{array}$ & $41.3 \pm 17.9$ & $41.3 \pm 18.4$ & $40.9 \pm 10.9$ & 0.890 \\
\hline \multicolumn{4}{|l|}{ Macroscopic type, $n(\%)$} & 0.048 \\
\hline Elevated & $54(21.1)$ & $45(19.1)$ & $9(42.9)$ & \\
\hline Flat & $47(18.4)$ & $45(19.1)$ & $2(9.5)$ & \\
\hline Depressed & $141(55.1)$ & $133(56.6)$ & $8(38.1)$ & \\
\hline Mixed & $14(5.5)$ & $12(5.1)$ & $2(9.5)$ & \\
\hline Tumor size $(\mathrm{cm})$, mean \pm SD & $3.2 \pm 2.0$ & $3.1 \pm 1.7$ & $4.8 \pm 3.1$ & 0.003 \\
\hline \multicolumn{4}{|l|}{ Histology, $n(\%)$} & 0.649 \\
\hline Differentiated & $157(61.3)$ & $143(60.9)$ & $14(66.7)$ & \\
\hline Undifferentiated & 99 (38.7) & $92(39.1)$ & $7(33.3)$ & \\
\hline \multicolumn{4}{|l|}{ Depth of invasion, $n(\%)$} & $<0.001$ \\
\hline$M$ & $94(36.7)$ & $94(40.0)$ & $0(0)$ & \\
\hline SM1 & $46(18.0)$ & $44(18.7)$ & $2(9.5)$ & \\
\hline SM2 & $43(16.8)$ & $36(15.3)$ & $7(33.3)$ & \\
\hline SM3 & $73(28.5)$ & $61(26.0)$ & $12(57.1)$ & \\
\hline \multicolumn{4}{|c|}{ Lymphovascular invasion, $n(\%)$} & $<0.001$ \\
\hline Negative & $213(83.2)$ & $204(86.8)$ & $9(42.9)$ & \\
\hline Positive & $43(16.8)$ & $31(13.2)$ & $12(57.1)$ & \\
\hline
\end{tabular}

$L N M$ lymph node metastasis, $M$ mucosal cancer, $S M 1$ submucosal invasion $<500 \mu \mathrm{m}$, SM2 submucosal invasion $500-1000 \mu \mathrm{m}, S M 3$ submucosal invasion $\geq 1000 \mu \mathrm{m}, S D$ standard deviation tumor size and invasion depths among the T1b gastric cancers without LVI (Table 3). In patients with SM1 (superficial submucosal invasion $<500 \mu \mathrm{m})$, there was no LNM in any tumor sized $\leq 3 \mathrm{~cm}$. However, in patients with SM2/3 (deep submucosal invasion $\geq 500 \mu \mathrm{m}), \mathrm{LNM}$ rate was $28.6 \%(2 / 7)$, even in the smallest tumor size group $(\leq 1 \mathrm{~cm})$.

Based on the criteria for endoscopic resection of early gastric cancers, no LNM was observed in the 113 patients satisfying the absolute or extended criteria (Table 4).

\section{Long-term survival}

The 5-year overall survival, disease-specific survival, and recurrence-free survival rates in Siewert type II/III early gastric cancers were $96.3 \%, 98.6 \%$, and $99.0 \%$, respectively.
O n comparing long-term survival in patients with Siewert II/III T1 cancers and matched non-cardia T1 cancers, the overall survival [hazard ratio (HR), 0.43, 95\% CI 0.18-1.01, $P=0.053$ ], disease-specific survival (HR $0.50,95 \% \mathrm{CI}$ 0.10-2.61, $P=0.409$ ), and recurrence-free survival (HR $0.61,95 \%$ CI $0.13-2.91, P=0.532$ ) did not differ significantly (Online Resource 3-5).

\section{Discussion}

In this study, we determined tumor size and LVI as the predictors of LNM in patients with T1b Siewert type II/III gastric cancers. A risk prediction model for LNM was developed, which was validated using bootstrap resampling. As it 
Table 2 Logistic regression analysis in T1b Siewert II/III cancer

\begin{tabular}{|c|c|c|c|c|}
\hline & \multicolumn{2}{|l|}{ Univariate analysis } & \multicolumn{2}{|c|}{ Multivariate analysis } \\
\hline & OR $(95 \% \mathrm{CI})$ & $P$ value & OR $(95 \% \mathrm{CI})$ & $P$ value $^{\mathrm{a}}$ \\
\hline Age & $0.97(0.93-1.02)$ & 0.278 & & \\
\hline Sex & & 0.883 & & \\
\hline Male & 1.00 & & & \\
\hline Female & $1.09(0.34-3.52)$ & & & \\
\hline Macroscopic type & & 0.237 & & \\
\hline Elevated or flat & 1.00 & & & \\
\hline Depressed & $0.57(0.23-1.44)$ & & & \\
\hline Tumor size & $1.43(1.14-1.78)$ & 0.002 & $1.42(1.10-1.82)$ & 0.007 \\
\hline Histology & & 0.662 & & \\
\hline Differentiated & 1.00 & & & \\
\hline Undifferentiated & $0.81(0.31-2.12)$ & & & \\
\hline Depth of invasion & & 0.056 & & \\
\hline SM1 & 1.00 & & & \\
\hline $\mathrm{SM} 2 / 3$ & $4.31(0.96-19.31)$ & & & \\
\hline Lymphovascular invasion & & $<0.001$ & & 0.002 \\
\hline Negative & 1.00 & & 1.00 & \\
\hline Positive & $5.63(2.15-14.71)$ & & $5.13(1.88-14.06)$ & \\
\hline
\end{tabular}

$S M 1$ superficial submucosal invasion $<500 \mu \mathrm{m}, S M 2 / 3$ deep submucosal invasion $\geq 500 \mu \mathrm{m}, O R$ odds ratio, CI confidence interval

${ }^{\text {a }}$ Backward selection method was applied (significance criteria 0.05 for stay)

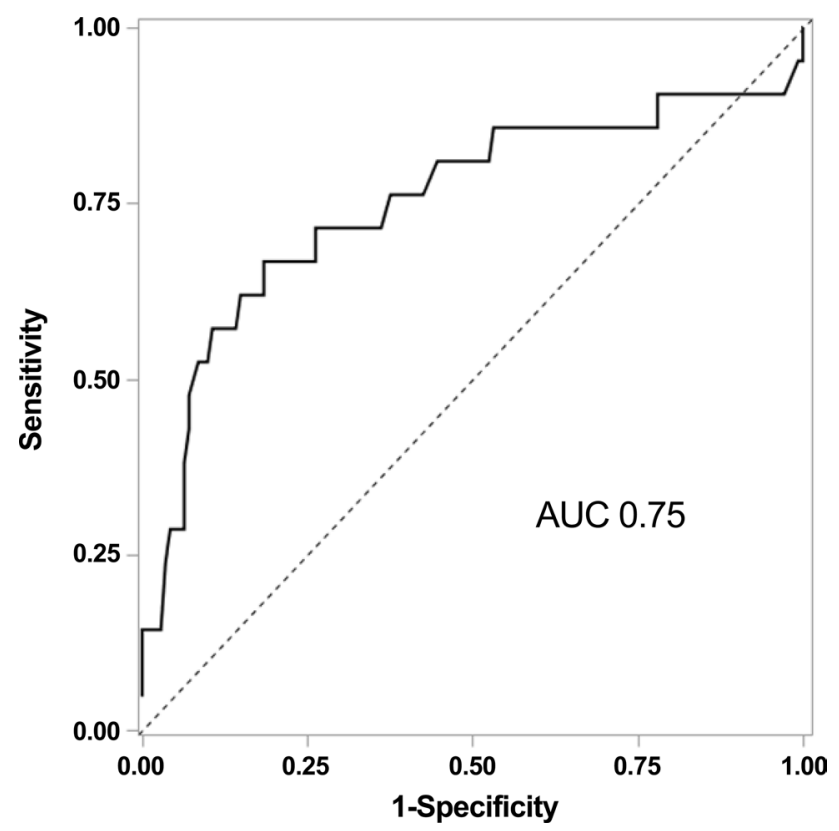

Fig. 1 Receiver operating characteristic curve analysis of the predicted probabilities of the multivariate logistic regression model in the presence of lymph node metastasis. AUC area under the curve

is generally accepted that patients with LVI should undergo surgery, we searched for the group with a negligible risk of LNM among patients without LVI, which were mucosaconfined cancers, or SM1 cancers with tumors $\leq 3 \mathrm{~cm}$ in

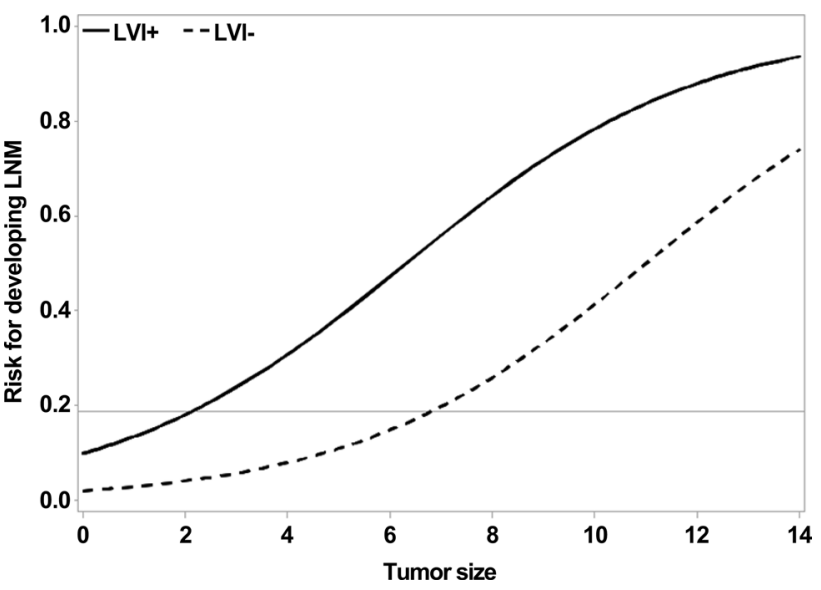

Fig. 2 Risk of lymph node metastasis as a function of tumor size and presence of lymphovascular invasion. $L N M$ lymph node metastasis, LVI lymphovascular invasion

size. Moreover, the LNM and long-term survivals of patients with T1 Siewert gastric cancer were not significantly different with matched non-cardia cancer patients. Therefore, the same expanded indication for endoscopic resection can also be applied to patients with Siewert type II/III.

Previous studies regarding the endoscopic resection of EGJ or cardia cancers generally evaluated safety and efficacy based on the curative resection rates, procedure-related adverse events, or local recurrence using 


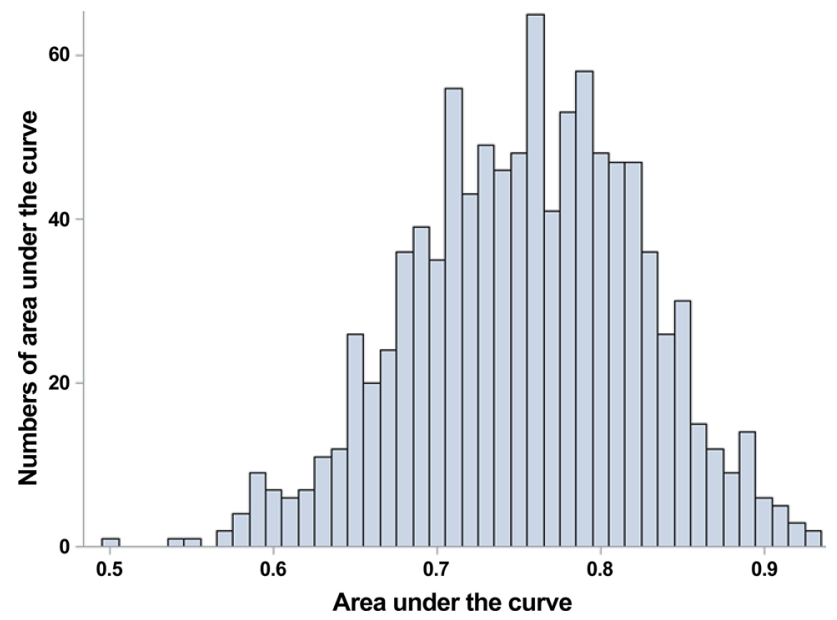

Fig. 3 Validation of the predicted model based on 1000 bootstrap samples

endoscopically resected tumors [18-21]. This method limits the evaluation of risks associated with lymph node involvement, which is a strong prognostic predictor for survival [22]. In our study, we evaluated the lymph node involvement in patients who underwent surgery for T1 gastric cancers of Siewert type II/III, and developed a risk prediction model for LNM that incorporated the tumor size and LVI status, which showed adequate discrimination. To the best of our knowledge, this is the first study to evaluate the lymph node patterns in early Siewert type II/III cancers.

Gastric cardia cancer appears more aggressive and consequently has a worse long-term prognosis than non-cardia cancers. Studies reported a higher incidence of LNM in cardia cancer when compared to non-cardia cancer [6, 23]. A systematic review of publications investigating gastric cardia cancer demonstrated that patients with locoregional LNM had the longest survival, with survival rates decreasing with distant LNM [24]. Furthermore, Saito et al. demonstrated a significantly higher LNM and worse 5-year survival in cardia cancer, compared with other sites. The lymph node and hematological recurrence rates were also higher in cardia cancers than other sites [6]. Moreover, Chen et al. used a semi-parametric method to demonstrate that, among patients with gastric cancer, adenocarcinomas of the cardia have shorter life expectancies and more expected years of life lost [25]. Despite the aggressive potential of gastric cardia cancers compared to non-cardia cancers, the risk of LNM and long-term survival in the early stages of the disease do not differ significantly. Although the reasons for such behavior are unclear, several mechanisms have been proposed. The preferential spread pattern of cardia cancer involves the locoregional gastric lymph node stations early followed by invading distal lymph nodes, which will affect the chance of survival [24]. In other words, early stages of gastric with only locoregional LNM may have a good prognosis regardless of location, but advanced cardia cancer that has signs
Table 3 Lymph node metastasis rate, according to tumor size, in lymphovascular invasionnegative T1b Siewert II/III cancer

\begin{tabular}{llllll}
\hline $\begin{array}{lllll}\text { Tumor size } \\
(\mathrm{cm})\end{array}$ & $\mathrm{SM} 1$ & & \multicolumn{3}{l}{ SM2/3 } \\
\cline { 2 - 3 } & Each group $(n, \%)$ & Accumulation $(n, \%)$ & & Each group $(n, \%)$ & Accumulation $(n, \%)$ \\
\hline$\leq 1$ & $0 / 2(0)$ & $0 / 2(0)$ & $2 / 7(28.6)$ & $2 / 7(28.6)$ \\
$1-2$ & $0 / 7(0)$ & $0 / 9(0)$ & $0 / 23(0)$ & $2 / 30(6.7)$ \\
$2-3$ & $0 / 10(0)$ & $0 / 19(0)$ & $2 / 30(6.7)$ & $4 / 60(6.7)$ \\
$3-4$ & $1 / 14(7.1)$ & $1 / 33(3.0)$ & $6 / 27(22.2)$ & $10 / 87(11.5)$ \\
$>4$ & $2 / 13(15.4)$ & $3 / 46(6.5)$ & $9 / 29(15.8)$ & $19 / 116(16.4)$ \\
\hline
\end{tabular}

$S M 1$ superficial submucosal invasion $<500 \mu \mathrm{m}, S M 2 / 3$ deep submucosal invasion $\geq 500 \mu \mathrm{m}$

\begin{tabular}{|c|c|c|c|c|c|c|c|}
\hline & \multicolumn{4}{|c|}{ Mucosal cancer } & \multicolumn{3}{|c|}{ Submucosal cancer } \\
\hline & \multicolumn{2}{|l|}{$\mathrm{UL}(-)$} & \multicolumn{2}{|l|}{$\mathrm{UL}(+)$} & \multicolumn{2}{|l|}{ SM1 } & \multirow{2}{*}{$\begin{array}{l}\text { SM2/3 } \\
\text { Any size }\end{array}$} \\
\hline & $\leq 2 \mathrm{~cm}$ & $>2 \mathrm{~cm}$ & $\leq 3 \mathrm{~cm}$ & $>3 \mathrm{~cm}$ & $\leq 3 \mathrm{~cm}$ & $>3 \mathrm{~cm}$ & \\
\hline $\begin{array}{l}\text { Differen- } \\
\text { tiated } \\
(n \%, \\
95 \% \mathrm{CI})\end{array}$ & 0/94 (0) & & & & $0 / 19(0)$ & $1 / 20(5.0,0.0-14.6)$ & $13 / 70(18.6,9.5-27.7)$ \\
\hline $\begin{array}{l}\text { Undiffer- } \\
\text { entiated } \\
(n \%, \\
95 \% \mathrm{CI})\end{array}$ & & & & & & $1 / 7(14.3,0.0-40.2)$ & $6 / 46(13.0,3.3-22.8)$ \\
\hline
\end{tabular}

LVI lymphovascular invasion, $U L$ ulcer, SM1 submucosal invasion $<500 \mu \mathrm{m}$ 
of distant LNM, which is a strong indicator of clinical outcome [26], may have a significantly worse prognosis than non-cardia cancers. Furthermore, the location of cardia cancers is associated with a higher incidence of postoperative morbidity and mortality, especially in the elderly or patients with other comorbidities who might have limited treatment options in advanced stages of the disease, leading to worse prognosis. Also, it is easier to miss the early stages of the cancer during screening endoscopy in this location, with the result that the cancer is discovered at a later and more advanced stage.

The poor prognosis reported in patients with Siewert type II/III gastric cancers raises the question as to whether the same endoscopic resection criteria for early gastric cancer can be applied. Our data of 256 patients with Siewert II/ III gastric cancer demonstrated that there were no cases of LNM according to the absolute and extended criteria for endoscopic resection of early gastric cancers (Table 4). Furthermore, we aimed to identify the group of patients with negligible LNM who were potentially suitable to undergo endoscopic resection, which were mucosa-confined tumors or SM1 cancers with tumor sizes $\leq 3 \mathrm{~cm}$, without LVI. Therefore, despite the poor prognosis of proximal gastric cancer, the same criteria can be applied for endoscopic resection of Siewert type II/III early gastric cancer. A stricter indication may not be necessary. It may also be reasonable to explore close observation, without additional treatment, according to the histopathological findings of the resected specimen. This rationale could be especially applicable to elderly patients with comorbidities, averting surgical risk and improving quality of life by preserving the gastrointestinal tract. Furthermore, considering the difficulties associated with accurate preoperative diagnosis caused by a narrow lumen and sharp angles in the cardia, the prediction model from this work is suitable as a staging technique to evaluate the need for additional surgery $[2,20]$.

Our study had several inherent limitations. First, although the majority of patients underwent D2 or D2+ lymphadenectomy, this study was not a randomized control study and the extent of lymphadenectomy was not consistent in all patients. Therefore, nodal metastases could have been underestimated in some patients, leading to a selection bias. Second, our study only used conventional hematoxylin and eosin staining, and therefore lymph node micrometastasis was difficult to diagnose. Molecular methods, including immunohistochemistry and reverse transcription-polymerase chain reaction, are needed for the accurate diagnosis of micrometastasis [27].

In conclusion, we proposed a risk prediction model for LNM based on tumor size and LVI status in early Siewert type II/III tumors, and demonstrated that the same expanded indication for endoscopic resection can be used. These results may be useful in clinical practice for deciding which patients should undergo endoscopic resection, as well as for staging to evaluate the need for additional surgery.

\section{Compliance with ethical standards}

Conflict of interest There are no conflicts of interest to declare.

Ethical approval All procedures followed were in accordance with the ethical standards of the responsible committee on human experimentation (institutional and national) and with the Helsinki Declaration of 1964 and later versions. Because of the retrospective nature of the study, the requirement for informed consent was waived.

\section{References}

1. Oda I, Abe S, Kusano C, Suzuki H, Nonaka S, Yoshinaga S, et al. Correlation between endoscopic macroscopic type and invasion depth for early esophagogastric junction adenocarcinomas. Gastric Cancer. 2011;14:22-7.

2. Griffin SM, Burt AD, Jennings NA. Lymph node metastasis in early esophageal adenocarcinoma. Ann Surg. 2011;254:731-6 (discussion 6-7).

3. Shah MA, Kelsen DP. Gastric cancer: a primer on the epidemiology and biology of the disease and an overview of the medical management of advanced disease. J Natl Compr Canc Netw. 2010;8:437-47.

4. McColl KE, Going JJ. Aetiology and classification of adenocarcinoma of the gastro-oesophageal junction/cardia. Gut. 2010;59:282-4.

5. Petrelli F, Ghidini M, Barni S, Steccanella F, Sgroi G, Passalacqua $\mathrm{R}$, et al (2017) Prognostic role of primary tumor location in nonmetastatic gastric cancer: a systematic review and meta-analysis of 50 studies. Ann Surg Oncol 24(9):2655-68.

6. Saito H, Fukumoto Y, Osaki T, Fukuda K, Tatebe S, Tsujitani $\mathrm{S}$, et al. Distinct recurrence pattern and outcome of adenocarcinoma of the gastric cardia in comparison with carcinoma of other regions of the stomach. World J Surg. 2006;30:1864-9.

7. Ychou M, Boige V, Pignon JP, Conroy T, Bouche O, Lebreton G, et al. Perioperative chemotherapy compared with surgery alone for resectable gastroesophageal adenocarcinoma: an FNCLCC and FFCD multicenter phase III trial. J Clin Oncol. 2011;29:1715-21.

8. Siewert JR, Stein HJ. Carcinoma of the cardia: carcinoma of the gastroesophageal junction: classification, pathology and extent of resection. Dis Esophagus. 1996;9:173-82.

9. Biondi A, Hyung WJ. Seventh edition of TNM classification for gastric cancer. J Clin Oncol. 2011;29:4338-9 (author reply 40-42).

10. Goto H, Tokunaga M, Miki Y, Makuuchi R, Sugisawa N, Tanizawa $\mathrm{Y}$, et al. The optimal extent of lymph node dissection for adenocarcinoma of the esophagogastric junction differs between Siewert type II and Siewert type III patients. Gastric Cancer. 2015;18:375-81.

11. Gotoda T. Endoscopic resection of early gastric cancer. Gastric Cancer. 2007;10:1-11.

12. Gong EJ, Kim DH, Ahn JY, Jung KW, Lee JH, Choi KD, et al. Comparison of long-term outcomes of endoscopic submucosal dissection and surgery for esophagogastric junction adenocarcinoma. Gastric Cancer. 2017;20:84-91.

13. Pyo JH, Lee H, Min BH, Lee JH, Choi MG, Lee JH, et al. Longterm outcome of endoscopic resection vs. surgery for early gastric cancer: a non-inferiority-matched cohort study. Am J Gastroenterol. 2016;111:240-9. 
14. Siewert JR, Stein HJ. Classification of adenocarcinoma of the oesophagogastric junction. Br J Surg. 1998;85:1457-9.

15. Japanese Gastric Cancer Association. Japanese gastric cancer treatment guidelines (ver. 4). Gastric Cancer. 2014;2017(20):1-19.

16. Kim HH, Hyung WJ, Cho GS, Kim MC, Han SU, Kim W, et al. Morbidity and mortality of laparoscopic gastrectomy versus open gastrectomy for gastric cancer: an interim report: a phase III multicenter, prospective, randomized Trial (KLASS Trial). Ann Surg. 2010;251:417-20.

17. AJCC. Cancer staging manual. 7th ed. New York: Springer; 2010.

18. Hirasawa K, Kokawa A, Oka H, Yahara S, Sasaki T, Nozawa A, et al. Superficial adenocarcinoma of the esophagogastric junction: long-term results of endoscopic submucosal dissection. Gastrointest Endosc. 2010;72:960-6.

19. Hoteya S, Matsui A, Iizuka T, Kikuchi D, Yamada A, Yamashita $\mathrm{S}$, et al. Comparison of the clinicopathological characteristics and results of endoscopic submucosal dissection for esophagogastric junction and non-junctional cancers. Digestion. 2013;87:29-33.

20. Omae M, Fujisaki J, Horiuchi Y, Yoshizawa N, Matsuo Y, Kubota $\mathrm{M}$, et al. Safety, efficacy, and long-term outcomes for endoscopic submucosal dissection of early esophagogastric junction cancer. Gastric Cancer. 2013;16:147-54.

21. Yamada M, Oda I, Nonaka S, Suzuki H, Yoshinaga S, Taniguchi $\mathrm{H}$, et al. Long-term outcome of endoscopic resection of superficial adenocarcinoma of the esophagogastric junction. Endoscopy. 2013;45:992-6.

22. Zhang M, Li Z, Ma Y, Zhu G, Zhang H, Xue Y. Prognostic predictors of patients with carcinoma of the gastric cardia. Hepatogastroenterology. 2012;59:930-3.

23. Maeda H, Okabayashi T, Nishimori I, Sugimoto T, Namikawa T, Dabanaka K, et al. Clinicopathologic features of adenocarcinoma at the gastric cardia: is it different from distal cancer of the stomach? J Am Coll Surg. 2008;206:306-10.

24. Okholm C, Svendsen LB, Achiam MP. Status and prognosis of lymph node metastasis in patients with cardia cancer: a systematic review. Surg Oncol. 2014;23:140-6.

25. Chen WY, Cheng HC, Wang JD, Sheu BS. Factors that affect life expectancy of patients with gastric adenocarcinoma. Clin Gastroenterol Hepatol. 2013;11:1595-600.

26. Yamashita H, Katai H, Morita S, Saka M, Taniguchi H, Fukagawa T. Optimal extent of lymph node dissection for Siewert type II esophagogastric junction carcinoma. Ann Surg. 2011;254:274-80.

27. Arigami T, Uenosono Y, Yanagita S, Nakajo A, Ishigami S, Okumura $\mathrm{H}$, et al. Clinical significance of lymph node micrometastasis in gastric cancer. Ann Surg Oncol. 2013;20:515-21. 